\title{
Le flâneur comme lecteur de la ville contemporaine
}

\author{
Anna Borisenkova \\ Professionnelle de recherche, chargée de cours, Université de Saint-Boniface \\ Maître de recherche, Centre for Fundamental Sociology, \\ National Research University Higher School of Economics \\ Address: avenue de la Cathédrale, 200, Winnipeg, Canada $\mathrm{R}_{2} \mathrm{H}_{\mathrm{OH}}$ \\ E-mail: ann.borisenkova@gmail.com
}

\begin{abstract}
The topic of the lover of street-life, the observer, the urban stroller and the wanderer, first appeared in Edgar Allan Poe's The Man of the Crowd. However, it was Charles Baudelaire who introduced the concept of the flâneur to the humanities and later, owing to Walter Benjamin, the flâneur was established as a central figure of modernity. Recent studies in the humanities and the social sciences have renewed discussions about the flâneur and the urban phenomenon of the flânerie. The roles of the flâneur in a contemporary city are described with the help of the two criteria: the degree of involvement of the flâneur in the urban environment and her capacity to transform the meaning of urban space. According to these criteria, we can distinguish the flaneurs who are active researchers of the city and those who participate in political activities. On the other side, there are more passive observers of the city life and consumers who aimlessly walk in postmodern shopping malls. The aim of the article is to analyze the evolution of theories of the flânerie and introduce an alternative approach to the concept. The approach is based on the hermeneutics of Paul Ricœur that views the flâneur as an active reader, actor-user of public space, and constructor of collective memory.
\end{abstract}

Keywords : flâneur, urban space, reader, memory, narrative, public space

Le concept du flâneur, comme "peintre de la vie ", demeure aujourd'hui un sujet de grand intérêt en sciences humaines, en sciences sociales, ainsi que dans le domaine des études urbaines (Wrigley, 2016 ; Bankovskaya, 2014 ; Shin, 2014 ; Merzeau, 2014). On remarque que l'expérience de cet(te) ami(e) de la rue, marchant dans les villes sans raison apparente, observant tout ce qui se passe dans les quartiers et les grands boulevards, tout en portant une attention particulière aux rythmes de la vie urbaine, nous expose bien en quoi peut consister l'expérience esthétique de la ville. Ce promeneur solitaire, ce flâneur attentif, se révèle être, à sa manière, un artiste de la rue, capable d'exercer plusieurs talents, soit écrivain, soit architecte de son parcours. De même, Charles Baudelaire décrit-il le flâneur comme « le peintre de la vie moderne » (Baudelaire, 1885). Ici, Baudelaire compare le flâneur à un type d'individus, dans le monde des artistes, «qui vont au musée du Louvre, passent rapidement, et sans leur accorder un regard, devant une foule de tableaux très intéressants, puis sortent satisfaits, plus d'un se disant : « Je connais mon musée » (Baudelaire, 1885: 52).

(C) Borisenkova A., 2017

(C) Centre for Fundamental Sociology, 2017 DOI: $10.17323 / 1728-192 \mathrm{X}-2017-2-75-88$ 
Dans cet article nous exposerons, dans un premier lieu, les diverses transformations subies par le concept de "flâneur », parmi les disciplines philosophique et sociologique. En second lieu, nous nous pencherons sur les caractéristiques qui peuvent être employées aujourd'hui pour décrire le flâneur postmoderne. Nous examinerons, en cela, les nombreux rôles du flâneur qui correspondent aux transformations changeantes du milieu urbain. Les rôles du flâneur, que l'on peut observer dans les villes, à partir du XIX siècle à nos jours, varient selon les deux critères suivants : la participation du flâneur dans le milieu urbain, ainsi que sa capacité de reconfigurer le sens de l'espace social. Il nous est possible d'abord de distinguer deux types de flâneurs urbains. Nous pouvons identifier un premier type, qui serait plutôt passif et qui ne contribue pas à la transformation du milieu social. Le flâneur correspond, dans ce cas-ci à, un simple observateur gardant une distance à l'endroit des enjeux sociaux et à un consommateur. À l'opposé de ce type, un autre existe, qui nous semblera plus actif, et que l'on peut reconnaître dans les rôles des chercheurs de la ville, ou des citoyens participant activement aux débats politiques municipaux. Alors que deux modèles du flâneur existent, comme nous venons de le voir, et qui sont entre eux diamétralement différents je propose, dans le présent article, de m’intéresser exclusivement au second modèle du flâneur, soit le rôle du lecteur de l'espace urbain. Pour conduire mon analyse, je vais appliquer l'approche herméneutique de Paul Ricœur qui est sous-estimée dans le cadre des études s’intéressant à la flânerie.

Il faut préciser que Ricœur n'a pas écrit beaucoup d'œuvres directement liées aux études urbaines. Ainsi, nous n’avons trouvé que deux travaux traitant de ce sujet: "Architecture et narrativité ", ainsi que " Propos d'un flâneur » (Ricœur, 1998, 2000). Néanmoins, ces deux essais, à eux seuls, nous permettent d'envisager une conception théorique alternative aux théories du flâneur existant dans les travaux classiques et dans les travaux sociologiques contemporains. En effet, Ricœur se distingue des approches classiques en proposant une image du flâneur où celui-ci est un acteur-utilisateur actif de l'espace public ; le flâneur est en ce sens, un lecteur actif de la ville et un constructeur de la mémoire collective. Nous nous intéressons, dans les prochaines pages, aux fonctions du flâneur contemporain comme constructeur de mémoire, pour comprendre son rôle dans lélaboration des espaces collectifs des villes d'aujourd'hui.

\section{Le peintre de la vie moderne : les origines du concept}

Le concept du flâneur a été introduit en sciences humaines et sociales par Walter Benjamin qui, à son tour, fait référence à Charles Baudelaire. Ces deux auteurs reconnaissent toutefois ensemble s'inspirer de la tradition anglaise, et plus particulièrement de l'oeuvre d'Edgar Poe, où sont décrites, tel qu'on le retrouve dans la nouvelle « The Man of the Crowd » les rues de Londres (Coverley, 2010). Poe fut le premier à décrire la foule comme étant le symbole de l'apparition de la ville moderne et de l'anonymisation de son habitant. C'est ainsi grâce à la lecture de Poe, effectuée par Baudelaire, que le flâneur est devenu l'image de l'observateur de la ville européenne. Dès lors, ce ne fut plus Londres, mais plutôt Paris, cette-grande ville de passages, qui devint l'habitat naturel du flâneur. 
Baudelaire souligne que le flâneur aime « la beauté générale qui est exprimée par les poètes et les artistes classiques " (Baudelaire, 1885: 52). Cependant, le flâneur n'est pas nécessairement un artiste. Baudelaire le décrit comme un homme singulier, un homme du monde et un homme des foules. Le point de départ du génie du flâneur est la curiosité. Le flâneur est curieux de tout ce qui se passe autour de lui, il est un observateur attentif. Pour cette curiosité, Baudelaire le nomme un "homme-enfant », un homme : "Possédant à chaque minute le génie de l'enfance, c'est-à-dire un génie pour lequel aucun aspect de la vie n’est émoussé » (Baudelaire, 1885:62). Baudelaire renonce à identifier le flâneur avec un autre type social de son époque : un dandy. Pour Baudelaire, le mot « dandy » implique une quintessence de caractère et une intelligence subtile, mais en même temps, le dandysme est caractérisé par une insensibilité et une attitude blasée. Par contre, le flâneur est amoureux de la vie, il expérimente une immense jouissance en regardant les mouvements de la ville et en écoutant ses rythmes. Une des caractéristiques très importantes du flâneur est ses relations ambivalentes avec la foule. Baudelaire écrit :

La foule est son domaine, comme l'air est celui de l'oiseau, comme l'eau celui du poisson. Sa passion et sa profession, c'est d'épouser la foule. Pour le parfait flâneur, pour l'observateur passionné, c'est une immense jouissance que d'élire domicile dans le nombre, dans l'ondoyant dans le mouvement, dans le fugitif et l'infini. Etre hors de chez soi, et pourtant se sentir partout chez soi ; voir le monde, être au centre du monde et rester caché au monde, tels sont quelques-uns des moindres plaisirs de ces esprits indépendants, passionnés, impartiaux, que la langue ne peut que maladroitement définir. L'observateur est un prince qui jouit partout de son incognito. (Baudelaire, $1885: 64$ )

Ainsi donc, le flâneur se mêle avec la foule tout en cherchant sa solitude en se détachant des autres. La référence de Baudelaire au sujet de la foule n'est pas accidentelle. En effet, la foule, selon lui, symbolise l'époque de la modernité. La ville de Paris décrite par Baudelaire [est] Paris du Seconde Empire, où Napoléon III régnait en tant qu'empereur, soit la période de 1852 à 1870, entre les Deuxième et Troisième Républiques. C'est dans cette période que Paris fut transformée en la capitale moderne. Cette nouvelle ville attira une libre circulation des marchandises et des personnes. À cette époque, le nombre de passagers d’omnibus augmenta jusquà 36 million en 1855 et 110 million en 1860 (Grotta, 2015 : 4). Afin d'expliquer ce nouveau phénomène d'urbanisation, plusieurs chercheurs se tournent vers une nouvelle intimité caractérisant la vie urbaine. Cette intimité est maintenant différente de l'intimité familiale ou des groupes d'affinités. Il s'agit de relations avec des étrangers : des personnes que nous ne connaissons pas.

Dans cet article, on utilise la notion du flâneur au masculin en suivant les travaux de Baudelaire et Benjamin. En même temps, on ne vise pas à indiquer un sexe masculin du flâneur, parce quon n’entend pas par là une personne physique, mais un regard spécifique. Par contre, Laura Elkin (2017) souligne l'importance du rôle de la flâneuse dans la ville. Elkin note que le terme «flâneuse " n'existe pas techniquement dans la langue française. Elle explique cette pensée, par le fait que les femmes de la ville répondent à l'image 
véhiculée dans les médias. En effet, on dit que ces habitantes marchent dans les villes plus souvent avec un but, elles jouent toujours leurs rôles sociaux de mère, de femme au foyer, d'employée. Ainsi, la flâneuse est une femme qui résiste aux normes de la société, elle est un objet d'observation par les hommes, mais cependant considérée comme une actrice active (Elkin, 2017). Tout en étant d'accord avec Elkin, nous utiliserons toutefois le terme classique de "flâneur ", sans lui reconnaître nécessairement un sexe défini, alors que le terme ne doit renvoyer ici qu'au type social de la personne adoptant la posture d'observateur de la vie urbaine.

Walter Benjamin, en introduisant le concept du flâneur, se tourne aussi vers la problématique des formes de vie nouvelle et des transformations économiques et techniques qui caractérisent le Paris hausmannien. En effet, ce sont ces lieux où les vieux quartiers sont remplacés par des grands boulevards, des cafés et des vitrines de grands magasins. Afin d'expliquer l'apparition du phénomène de la flânerie, Benjamin adresse la question d'une nouvelle architecture de son époque.

Il observe que l'impérialisme napoléonien favorise le capitalisme de la finance. Il note qu’à la Cité, berceau de la ville, «il n'y restait qu'une église, un hôpital, un bâtiment public et une caserne " (Benjamin, 1939:17). Une remarque très importante de Benjamin est que « le véritable but des travaux de Haussmann était d'assurer qu'il n’y ait pas de guerre civile. Il voulait rendre impossible à tout jamais la construction de barricades dans les rues de Paris »(Benjamin, 1939 : 17). Ainsi, la nouvelle architecture tend à bloquer la socialité dans la ville. Comme Benjamin le note « les habitants de la ville ne s'y sentent plus chez eux ; ils commencent à prendre conscience du caractère inhumain de la grande ville» (Benjamin, $1939: 17)$.

À cet égard, Benjamin fait référence à Charles Fourier concernant le canon architectonique du phalanstère qui a caractérisé ce Paris moderne. Pour Fourier, les phalanstères et les ensembles de bâtiments à usage communautaire formeront le socle d'un nouvel État. Benjamin écrit : "Les passages qui se sont trouvés primitivement servir à des fins commerciales, deviennent chez Fourier des maisons d'habitation. Le phalanstère est une ville faite de passages... Dans cette "ville en passages" la construction de l'ingénieur affecte un caractère de fantasmagorie. La "ville en passages" est un songe qui flattera le regard des parisiens jusque bien avant dans la seconde moitié du siècle... La ville y adopte une structure qui fait d’elle avec ses magasins et ses appartements le décor idéal pour le flâneur » (Benjamin, 1939: 8).

Notamment, le flâneur est né dans une ville de passages qui devient son intérieur. Toutefois, selon Benjamin, le flâneur ne se sent pas chez lui dans la ville. Il n'est pas attaché à un lieu ou à un groupe social. Georg Simmel dans «The Metropolis and Mental Life " adresse les conditions de la grande ville qui causent aliénation dans la consommation et la superficialité des contacts humains (Simmel, 2002). Chez Simmel, le citadin obsédé par des stimulations spécifiquement urbaines ne perçoit plus les différences qualitatives. Il devient blasé. Lanalyse post-simmelienne de l'interaction de l'individu avec l'environnement urbain se retrouve dans la sociologie de l'École de Chicago. Par exemple, Robert Park remarque la rationalité et l'aliénation de l'habitant dans des grandes villes 
américaines telles que Chicago. C'est ici, dans l'environnement très dynamique et changeant qu'on trouve les origines du « sujet névrose urbain » qui est indiffèrent face à tous ce qui se passe dans la ville (Park, 1915).

Par contre, selon Benjamin, en marchant dans les rues, le flâneur observe attentivement des événements urbains. En décrivant le portrait du flâneur, Benjamin souligne deux traits principaux. Premièrement, le flâneur est un explorateur de la ville sociale. Benjamin écrit : "Dans la personne du flâneur l'intelligence se familiarise avec le marché » (Benjamin, 1939 : 14). Pourtant, selon Benjamin, le flâneur n’a pas de position économique, ni de position politique dans la société moderne. Il est simplement choqué par le milieu urbain et la technologie qui changent très rapidement. C'est pour cette raison que, note Benjamin, «le flâneur cherche un refuge dans la foule. La foule est le voile à travers lequel la ville familière se meut pour le flâneur en fantasmagorie. Cette fantasmagorie, où elle apparaît tantôt comme un paysage, tantôt comme une chambre, semble avoir inspiré par la suite le décor des grands magasins, qui mettent ainsi la flânerie même au service de leur chiffre d'affaires » (Benjamin, 1939:14). Il est important de préciser quen parlant du flâneur, Benjamin n'entend pas par là une personne physique, mais un regard spécifique ; c'est un regard ou une perspective d'observateur prise par des habitants de la ville moderne.

Deuxièmement, pour Benjamin, le regard du flâneur est un regard mélancolique et allégorique. Benjamin explique : "Le regard que le génie allégorique plonge dans la ville trahit bien plutôt le sentiment d'une profonde aliénation. C'est là le regard d'un flâneur, dont le genre de vie dissimule derrière un mirage bienfaisant la détresse des habitants futurs de nos métropoles » (Benjamin, 1939: 14).

Ainsi, Benjamin décrit le flâneur comme une figure conciliant trois activités : la marche, l'observation et l'interprétation (Nuvolati, 2009), Toutefois, le sexe du flâneur, son apparence, son éducation et son statut social nont pas d'importance pour nous. Le concept du flâneur peut être défini, en premier lieu, comme une optique spécifique d'une personne marchant dans la ville. Cette optique est un produit de la société moderne caractérisée par l'aliénation et la solitude. Le flâneur représente un outil privilégié pour explorer la vie urbaine dans la métropole moderne et les rapports sociaux qui deviennent plus aliénants. Mais la question qu'on veut poser : Ce flâneur est-il capable de changer le milieu urbain et de reconfigurer le sens de l'espace?

Chez Baudelaire et Benjamin, on ne trouve pas de telles caractéristiques. Le flâneur comme acteur politique apparait dans les travaux d'André Breton et Louis Aragon : Nadja et Paris Peasant (Coverley, 2010). Ici l’observateur urbain résiste à la destruction et à la reconstruction de Paris par le projet d'Haussmann ainsi il participe aux mouvements politiques. Paris est décrite comme un lieu de la réclamation politique et les observateurs urbains qui occupent les rues et les places publiques deviennent des figures majeures de la résistance. La question sur la capacité du flâneur, de changer le milieu urbain, est posée d'une façon plus articulée dans les discussions autour de la ville contemporaine et postmoderne. 


\section{La figure du Flâner dans la ville contemporaine}

Comme on a déjà remarqué, le concept du flâneur se déclare comme un résultat de la modernisation de la société, son aliénation et la transformation de relations sociales. Les questions que je voudrais poser sont les suivantes : Comment le concept du flâneur pourrait-il être perçu aujourd'hui ? Quel sens obtient-il dans la société postmoderne?

Les sciences humaines et sociales du XXe siècle ont été marquées par l'intérêt envers les interactions de l'individu avec l'espace urbain qui l'entoure. Les questions telles que la dimension spatiale des relations humaines et la coexistence des gens dans l'espace limité deviennent les sujets de l'analyse profonde des penseurs sociaux. Par exemple, Michel Foucault propose l'emploie du concept d'hétérotopie, afin de décrire l'espace urbain contemporain : "Un espace qui est tout chargé de qualité, un espace, qui est peut-être aussi hanté de fantasme ; l'espace de notre perception première, celui de nos rêveries, celui de nos passions détiennent en eux-mêmes des qualités qui sont comme intrinsèques ; c'est un espace léger, éthéré, transparent, ou bien c'est un espace obscur, rocailleux, encombré... » (Foucault, 1967: 46) Selon Foucault, cette hétérotopie est caractérisée par les relations humaines diverses qui définissent des emplacements irréductibles les uns aux autres et absolument non superposables (Foucault, $1967: 46$ ). Comme le note la sociologue Svetlana Bankovskaya, la créativité de l'espace hétérotopique crée des conditions pour un mouvement quotidien sans but, c'est à dire, la flânerie (Bankovskaya, 2014).

La créativité de la ville et l'importance des pratiques quotidiennes sont soulignées aussi par Michel de Certeau qui compare la vue sur la ville de New York depuis le $110^{e}$ étage du World Trade Center et la vue de l'intérieur de la vie (De Certeau, 1990). Il décrit la première perspective ainsi : "La masse gigantesque s'immobilise sous les yeux. Elle se mue en texturologie où coïncident les extrêmes de l'ambition et de la dégradation, les oppositions brutales de races et de styles, les contrastes entre les buildings crées hier, mués déjà en poubelles et les irruptions urbaines du jour qui barrent lespace » (De Certeau, 1990 : 139). On ne peut pas comprendre la ville réelle en la regardant « en haut» parce qu’on ne peut voir « qu’un artefact optique » ou qu'un simulacre « théorique » (De Certeau, 1990 : 141). Par contre, c'est « en bas » que vivent les pratiquants ordinaires de la ville. Selon De Certeau, la forme élémentaire de cette expérience urbaine, «ils sont des marcheurs ", Wandersmänner, dont le corps obéit aux pleins et aux déliés d'un « texte " urbain qu'ils écrivent sans pouvoir le lire (De Certeau, 1990 : 141). La flânerie ici est le moyen fondamental d'habiter la ville, de lire son texte et de comprendre son histoire.

La marche, comme la pratique fondamentale de la vie urbaine, est analysée dans les travaux des situationnistes, tels que Guy Debord, Iain Sinclair, Peter Ackroyd, Stewart Home et Will Self, qui dans les années 1950, parlent de la psychogéographie, soit une approche qui se focalise sur les effets de l'environnement géographique sur des comportements et des émotions des individus (Coverley 2010 : 3). Les situationnistes imaginent un contexte dans lequel un individu, qui n’est pas nécessairement un chercheur, laisse de côté ses motifs, ses raisons, ses explications habituelles, et ce, pendant une certaine période de temps. L'individu subordonne alors son mouvement à ceux de la ville et c'est alors 
que l'environnement direct en vient à le dominer en le poussant à arrêter et à changer sa direction. Afin d'expliquer ce mouvement, Debord introduit le concept de "la dérive " qui se définit comme " une technique du passage hâtif à travers des ambiances variées. Le concept de dérive est indissolublement lié à la reconnaissance d'effets de nature psychogéographique, et à l'affirmation d'un comportement ludique-constructif, ce qui loppose en tous points aux notions classiques de voyage et de promenade » (Debord, 2017).

De récents travaux sociologiques ont accordé aussi un regain d'intérêt au flâneur. Pour Anthony Giddens, par exemple, le flâneur est le symbole de l'anonymat de l'espace urbain postmoderne (Giddens, 1991). Maintenant, le flâneur reflète l'incertitude de la société contemporaine, la mobilité des relations épisodiques et fragmentaires. À cet égard, il faut que nous examinions de nouveau le milieu du flâneur et son rôle. Tout d'abord, je voudrais considérer les deux caractéristiques de la ville postmoderne. La première est la consommation qui enveloppe les espaces publiques et historiques de la ville aujourd'hui. La deuxième caractéristique est une mobilité ainsi qu’une vitesse accélérée de la vie urbaine. Un autre sociologue, Zygmund Bauman, par contre, souligne le rôle du flâneur comme consommateur et joueur voyageant.

\section{Le Flaneur-consommateur}

Selon Zygmunt Bauman, la ville de passage, où le flâneur de Baudelaire et Benjamin est né, n'existe plus. Les passages aujourd'hui ne sont qu'attractions touristiques; ils restent conservés par l'industrie du patrimoine dans leur authenticité (Bauman, 1994 : 148). Les passages ne comprennent que la nostalgie du passé. Pour cette raison, Bauman compare la métropole avec un désert. Il dit : « La rue est un espace mort... Il est seulement un moyen de passage » (Bauman, $1994: 148)$ Dans les passages et grands boulevards il n’y a pas d'action. Les citadins marchent, traversent les rues sans arrêter, mais par rapport aux flâneurs, on remarque que les nouveaux citadins ne regardent pas autour deux. Pourtant, Bauman nous indique un nouveau milieu de la flânerie contemporaine - des centres commerciaux ou les " malls ». Par conséquent, les centres commerciaux, que Bauman nomme " Disneylands », deviennent l'intérieur des flâneurs. Le sociologue écrit : « Le flâneur d'hier se promenait sans but ; mais aujourd'hui, se trouve un but dans cette inutilité, il s'agit d'une fonction, d'une utilité, d'un design, qui nont rien du tout avoir avec la propre décision du flâneur» (Bauman, 1994: 150). En suivant les souvenirs de Dave Hill, Bauman utilise l'exemple de West Edmonton Mall à l'Alberta :

West Edmonton Mall n'est pas tellement un centre commercial, il est plutôt comme un fantasme de consommation entièrement intégré qui réussit à être stupidement mielleuse. Ce qui est d'ailleurs tout à fait ridicule et absolument hors de ce monde. Ce bâtiment est l'étude d'une science exacte et ce panorama intérieur est rien sinon l'état-of-the-art... Le plaisir est de faire partie d'un univers alternatif tranquillement fou où la mince ligne qui sépare le shopping du divertissement à la fin du XXe siècle devient presque totalement effacé. (Bauman, 1994 : 152) 
Ainsi, Bauman introduit un nouveau rôle du flâneur - un joueur et plus précisément, - joueur voyageant. Le flâneur toujours fait partie des jeux de " Disneylands », donc il devient un acheteur (acteur passif) préoccupé par la consommation. Le flâneur est cependant exproprié de son ancien rôle : le mystère n'existe plus, l'imagination et la réalité se croisent et la fantaisie du poète n’est plus nécessaire.

Un autre regard, très intéressant sociologiquement, sur la flânerie dans la ville contemporaine, est proposé par Jieun Shin dans son oeuvre "Le flâneur postmoderne ». Shin propose de voir le phénomène de la flânerie comme une forme de résistance silencieuse aux normes sociales existantes dans notre société. Premièrement, comme nous indique Shin, " vouloir être inutile est à l'opposé des valeurs établies dans la société moderne, dans l'actuel système économique marchand» (Shin, 2014 : 21). Le flâneur, dans la classique définition de Baudelaire, marche dans la ville sans but, de cette manière il soppose au rythme intense et des exigences de la ville contemporaine. Deuxièmement, dans l'époque de la mobilité automobile et post-automobile, la marche sans but est déjà une forme de résistance silencieuse. Comme Shin le dit, "L'homme contemporain a largement perdu sa naïveté pour suivre et s'adapter dans cette société de contrôle, tandis que le flâneur adopte un rythme lent, et gaspille le temps précieux comme l'or » (Shin, 2014 : 23).

Enfin, Shin renverse l'idée sur la solitude du flâneur. Selon Shin, le flâneur postmoderne ne cherche pas le refuge dans la foule mais, cependant, il soppose à toutes les personnes qui se cachent aujourd'hui dans leur bureau, leur maison, leur voiture et le métro. Le flâneur est libéré de la phobie du contact humain. De plus, en marchant dans la rue, l'individu solitaire peut trouver un contact avec les autres plus facilement. Shin ajoute que de cette manière le flâneur constitue un réseau avec les autres solitaires dans la rue, dans la foule; le Flash Mob en est l'exemple.

Il est intéressant de comparer le concept classique du flâneur de Baudelaire et de Benjamin, qui le décrivent chacun comme un chercheur et un peintre de la vie moderne, tandis que le flâneur postmoderne de Bauman est moins actif, il perd sa curiosité et n'est plus capable de changer son milieu urbain. Ses promenades dans les centres commerciaux signifient son époque de consommation et de globalisation. Toutefois, mon article vise à proposer une vision alternative du rôle du flâneur dans la ville contemporaine. Je vise à analyser le flâneur postmoderne autrement ; je propose de le considérer comme un acteur actif qui contribue à la vie urbaine.

\section{Acteur-utilisateur actif de l'espace public}

Une conception intéressante, relativement au phénomène de la flânerie, est proposée par les urbanistes Ash Amin et Nigel Trift. Selon ces auteurs, dans les villes en changement rapide, le flâneur, en tant qu'intellectuel vagabond, possède à la fois la sensibilité poétique et la science nécessaire pour lire la ville, brosser le portrait des multiples usages de ses rues et dépasser les stéréotypes. Le flâneur est capable de lire et interpréter la transitivité de la ville postmoderne. Selon Amin et Trift, la transitivité marque l'ouverture spatiale et temporelle de la ville, qui permet à la ville de se modeler et de se remodeler (Amin, Trift, 
2002 : 184). Pour les urbanistes, le flâneur n'est pas un naïf et un impressionnable dilettante, dont parle, par exemple, Zygmunt Bauman. Par contre, ce nouveau type du flâneur dispose d'une sensibilité et d'un langage lui servant à parler et à analyser des villes. Selon Amin et Trift, le meilleur exemple de la flânerie est le souvenir de Walter Benjamin luimême, lorsqu'il examinait la transitivité de Naples, Moscou et Marseille.

Ainsi donc, la figure du flâneur est un lecteur attentif et intellectuel de la ville. Ses lectures ne sont pas romantiques, mais, elles nous permettent plutôt de comprendre et d'expliquer les multiples usages de la ville. Sur le plan pratique, cela nous dit que les connaissances du flâneur peuvent nous aider à comprendre la transitivité de la vie urbaine concernant sa mobilité, ainsi que les changements quotidiens de sa population, sans oublier, les problèmes sociaux tels que l'inégalité, la pauvreté et le racisme. Bien évidemment, on ne conçoit pas la flânerie comme le seul moyen de la connaissance urbaine. Afin de répondre aux diverses questions sur la vie de la métropole postmoderne, on a besoin d'autres moyens comme des photos, des cartes routières, des guides historiques. Toutefois, les lectures sur le flâneur nous donnent une opportunité unique de connaitre la ville à travers une expérience personnalisée et vivante. Comment pourrait-on décrire la procédure de lecture de la ville par le flâneur? Qu'est-ce qu'il apporte dans la vie urbaine par sa lecture?

Une conception alternative du rôle du flâneur dans la ville contemporaine, peut aussi être tirée d'un cadre théorique plus solide, nous référant ici à l'approche herméneutique de Paul Ricœur. Tout d'abord, Ricœur propose d'examiner l'espace urbain comme un texte, en montrant un parallélisme étroit entre l'architecture et la narrativité. Dans son analyse, il suggère l'idée du parallélisme entre le construire et le raconter. Le philosophe inscrit cette analyse au sein des trois rubriques successivement développées dans son œuvre Temps et Récit, et qu'il avait placées sous le titre très ancien de mimesis : un stade de "préfiguration », celui où le récit avec tout son sens est engagé dans la vie quotidienne, dans la conversation, au stade d'un récit raconté qui était la « configuration » du sens du récit. Ricœur termine sa succession des rubriques par un stade de lecture et de relecture, la « réfiguration » (Ricœur, 1983).

Ricœur suit un mouvement parallèle du coté du construire : d'un stade de la "préfiguration » qui est lié à l'acte d'habiter à un deuxième stade de l'acte de construire, pour réserver finalement un troisième stade de « refiguration », la relecture de nos villes et de nos lieux d'habitation. Le parallélisme entre narrativité et architecture est expliqué par la remarque de Ricœur qu'au stade de la préfiguration « toute histoire de vie se déroule dans un espace de vie. L'inscription de l'action dans le cours des choses consiste à marquer l'espace d'événements qui affectent la disposition spatiale des choses " (Ricœur, 1998 : 5). Au stade de la configuration, l’euvre architecturale comme le texte est « un message polyphonique offert à une lecture à la fois englobante et analytique. Il en est de l'œuvre architecturale comme de la mise-en-intrigue, qui... ne rassemble pas seulement des événements, mais des points de vue, à titre de causes, de motifs et de hasards " (Ricœur, $1998: 9)$. 
En outre, Ricœur nous indique que la façon de lier l'espace et le temps de la ville est différente de la façon de lier l'espace et le temps de la maison. Pour lire la ville, le philosophe propose de considérer un cercle qui est aussi analogique du cercle du mimesis : construction, destruction et reconstruction. Ricœur note que « ce rythme est particulièrement approprié à certaines villes d'Europe affectées par les catastrophes du XX siècle, comme Rome, Berlin et Paris » (Ricœur, 2000 : 11).

De même, Ricœur nous rappelle la différence entre la configuration d'un bâtiment singulier (la maison, l'église, la banque) et la configuration de la ville : " La configuration d'une ville n'est jamais maitrisée par un seul homme, fût-il génial et tout puissant. Un projet urbain, - dit le philosophe, - même de grande envergure, s'insère dans une longue histoire qui échappe au contrôle d'une génération » (Ricœur, 2000 : 12). Par rapport à l'architecture, le projet urbain n'est jamais achevé. La ville peut être détruite partiellement ou complètement et sa reconstruction peut se faire à l'identique ou à nouveaux plans. Ricœur donne des exemples de Rome et de Berlin. Rome est l'idéal type de la construction continue et de l'accumulation des styles. À l’opposé de Rome, Berlin est une ville jamais construite, mais une ville détruite.

Un argument ricoeurien consiste à dire quavec la troisième composante de la mimesis, soit la lecture, le rapprochement entre le récit et l'espace urbain se fait plus étroit. Comme le lecteur vient au texte avec ses attentes propres, celles-ci peuvent être confrontées aux propositions de sens du texte dans la lecture. Le lecteur de l'espace urbain lit et relit les lieux de vie à partir de sa manière d'habiter. Ici Ricœur parle de l'habiter comme une réponse ou comme une riposte au construire. Le philosophe note : "L'habiter réceptif et actif implique une relecture attentive de l'environnement urbain, un réapprentissage continu de la juxtaposition des styles, et donc aussi des histoires de vie dont les monuments et tous les édifices portent la trace » (Ricœur, $2000: 15$ ).

Ainsi, chez Ricœur, le flâneur occupe le rôle de lecteur de l'espace urbain suivant les stades de la mimesis, en marchant dans la ville et en observant tout ce qui se passe autour de lui/elle.

Il faut souligner que pour Ricœur, l'action de lire peut devenir une action active : « Et la même palette de réponse que tout à l'heure peut être parcourue, de la réception passive, subie, indifférente, à la réception hostile courroucée - même celle de la Tour Eiffel, à son époque!» (Ricœur, 2000 : 15). Par rapport aux flâneurs de Benjamin, Bauman et Giddens, chez Ricœur, le lecteur de l'espace urbain peut contribuer au développement des espaces de vie en réagissant aux processus de construction et en donnant sa critique afin d'améliorer l'ambiance urbaine.

Par ailleurs, le lecteur de l'espace urbain fait un travail de mémoire. Ricœur nous indique que les lieux de vie sont les « lieux de mémoire » comprenant des « mémoires des époques différentes » (Ricœur, 2000 : 15). Pour Ricœur, le lecteur actif ne fait pas seulement le travail de la mémoire-répétition, pour laquelle rien ne vaut que le bien connu et le nouveau et odieux, mais il/elle manifeste sa capacité de faire le travail de la mémoire-reconstruction. Le principe de ce type de mémoire s'est basé sur l'idée que « le nouveau doit être accueilli avec curiosité et le souci de réorganiser l'ancien en vue de faire place à ce 
nouveau " (Ricœur, 2000 : 15). Ainsi, le flâneur, qui lit la ville, fait plus que la procédure de l'observation. Il défamiliarise le familier et familiarise le non-familier dans la ville, donc, en certaine manière, le flâneur contribue aussi aux changements de l'espace urbain.

Ici, le flâneur d'espace se fait flâneur d'histoire, il découvre l'étonnante tolérance des styles architecturaux. Il est important de remarquer que, selon Ricœur, "la fonction du flâneur curieux est de reconstituer le staccato de l'histoire d'une ville donnée en rythme binaire de la construction et la reconstructio " (Ricœur, $2000: 12$ ).

En même temps, Ricœur souligne un autre rôle important du flâneur contemporain, c'est apprendre l'espace urbain. Afin de décrire cette fonction, le philosophe donne un exemple du Musée Guggenheim à Bilbao : «À l'inverse des autres monuments commémoratifs, le musée planté en pleine ville se veut anticipateur d'une histoire à faire... Le monument ne parle qu’à un entendement abstrait, structuré par l'ordinateur ; il ne réveille aucun schématisme connu, façonné par l'histoire des styles et inscrit dans l'imaginaire collectif : vous ne retrouvez ni la colonne, ni l'architrave, ni le cube, ni la sphère, ni la pyramide, ni les courbes canoniques... C'est cela l'entendement nu, sans schématisme, sans imaginaire, sans structures imagées par une mémoi.re commune » (Ricœur, 2000 : 13). La fonction du flâneur ici n'est pas de reconnaitre, c'est d'apprendre. Le flâneur apprend des espaces inédits et, par ce moyen, ouvre une autre porte de la mémoire, celle de l'universel. Ainsi, le rôle du flâneur, dans ce contexte, n'est pas de reconstruire ou de reconnaître, mais d'apprendre les nouveaux symboles de la globalisation et les significations abstraites du présent et de l'avenir.

Il est important de souligner que la place du flâneur dans la pensée de Ricœur est différente de la position attribuée à létranger par Simmel, de même du flâneur de Benjamin. Pour Ricœur, le flâneur n'est ni aliéné ni indifférent. Au contraire, le flâneur observe et étudie l'environnement urbain et contribue aussi activement à des pratiques de commémoration et de deuil. Ricœur note que le flâneur fait non seulement interpréter l'histoire de la ville à travers la construction, la destruction et la reconstruction, mais aussi imagine la mémoire de l'habitant et tente de comprendre la ville du point de vue de ceux qui y vivent. D’ailleurs, Ricœur assigne un rôle important au flâneur : c'est à visiter les lieux qui ne sont pas fréquemment visités par les touristes, tels que les cimetières et ses sépultures qui témoignent des vivants de jadis. En disant cela, Ricœur nous indique un autre rôle du flâneur postmoderne : de "réinscrire les cimetières dans l'enceinte imaginaire de la ville, afin qu'elle inclue les vivants et les morts dans sa grande mémoire de pierre, de bois, de béton, de verre, de tous les métaux futuristes » (Ricœur, $2000: 13$ ).

Ainsi, le flâneur réinterprète la mémoire du passé et reconfigure la mémoire de l'espace habité. En raison de cet observateur réflexif, le passé, le présent et l'avenir deviennent unis en une seule image de la ville. Désormais, le flâneur ricoeurien démontre une autre qualité qui le rend unique. Le flâneur prend la responsabilité de la mémoire de la ville. Sa fonction est de découvrir les réponses aux questions du passé et de créer une image de la ville de l'avenir.

Est-ce que nous pouvons appliquer l'approche ricœurienne aux autres formes de la flânerie? 
Aujourd'hui nous parlons beaucoup de la cyber-flânerie, parce que plutôt que de sortir et de marcher, plusieurs personnes préfèrent passer leur temps sur Internet en surfant d'un site à l'autre. D'un côté, les réseaux sociaux permettent le " partage d'expériences flâneuses, par la diffusion de textes, de photos, de vidéos d’ouvres graphiques, de captages sonores qui rassemblent des flâneurs de tous les coins du monde autour de l'idée d'une meilleure conscience de son milieu " (Rioux Soucy, 2016). Ainsi, nous pouvons considérer l'espace virtuel comme le texte rempli des symboles et des sens qu'on peut lire, traduire et interpréter. De l'autre côté, comme le sociologue David Le Breton observe, «la flânerie, dans sa royauté, implique le corps, la sensorialité, les émotions, la curiosité, elle est une posture créative devant le monde qui laisse des traces de mémoire, suscite des rencontres. L'errance sur le Web, elle, dissout la présence dans une sorte de transe qui ne laisse aucune trace de mémoire, fait-il valoir » (Rioux Soucy, 2016). Le modèle du flâneur ricoeurien pourrait être appliqué, mais en même temps devrait être adapté aux réalités d'aujourd'hui. Comment peut-on « habiter » l'espace virtuel ? Est-ce qu'on peut parler de la mémoire de lespace sans aucunes traces physiques ? Ces questions sont des défis retrouvés dans des études contemporaines sur la flânerie.

\section{Conclusion}

La question que je voudrais poser finalement, est la suivante : Comment l'approche herméneutique transforme-t-elle l'idée de la flânerie aujourd'hui ? Il faut aussi dire que par rapport aux fondateurs de la théorie de la flânerie, Ricœur en analysant le rôle du flâneur, ne se focalise pas sur une époque particulière. Pour le philosophe, la figure du flâneur n'est pas un produit de son époque historique, soit moderne ou postmoderne. Par contre, le flâneur ricœurien se trouve hors du temps historique. Il est vrai que Ricœur décrit le flâneur comme un type social qui fait le travail de la mémoire, mais ce travail peut avoir lieu dans toutes les périodes de l'histoire. Ricœur ne relie pas le flâneur aux normes sociales : le flâneur ricœurien ne soutient ni les normes existantes dans la société de certaine période ni celles qui résiste à eux. En même temps, Ricœur souligne la contribution constructive de la flânerie à lenvironnement urbain et, par rapport à autres théoriciens, il nous offre une vision positive du flâneur urbain.

En suivant Ricœur, nous pouvons décrire le portrait du flâneur comme une personne très curieuse, attentive, ayant l'esprit avide de savoir. Toutefois, nous pouvons trouver les mêmes caractéristiques personnelles dans les descriptions de Baudelaire et Benjamin. En outre, dans ce portrait de qualité, Ricœur ajoute les traits qu'on ne trouve pas dans les travaux sociologiques contemporains et qui créent une image unique, c'est-à-dire la responsabilité du travail de la mémoire, la capacité de répondre aux défis de l’environnement urbain. Il faut aussi ajouter que le flâneur ricœurien possède une figure sociable, à l'inverse de l'individu blasé de Simmel et du flâneur classique qui cherche un refuge dans la foule. Le flâneur, dans l'approche herméneutique ricœurienne, est proche de l'habitant de la ville, et tente de comprendre celle-ci du point de vue de ceux qui y vivent. Nous 
pouvons donc conclure que le flâneur ricœurien est un utilisateur de l'espace urbain de l'avenir : curieux, actif et responsable.

\section{Bibliographie}

Ash A., Trift N. (2002) Cities: Reimagining the Urban, Cambridge: Polity Press.

Bankovskaya S. (2014) A Conception of, and Experiments with, "Heterotopia" as a Condition of Stable, Unpurposive, Everyday Movement. Basic Research Program Working Papers, Moscow: HSE.

Baudelaire Ch. (1885) Le Peintre de la vie moderne, Paris: Calmann Lévy.

Bauman Z. (1994) Desert Spectacular. The Flâneur (ed. K. Tester), London: Routledge, pp. $138-157$.

Benjamin W. (1939) Paris, capitale du XIXe siècle, 1939. Available at: http://classiques. uqac.ca/classiques/benjamin_walter/paris_capitale_19e_siecle/paris_capitale.html (accessed 10 June 2017).

De Certeau M. (1990) L'invention du quotidien. Arts de faire, Paris: Gallimard.

Debord G. (2017) Théorie de la dérive: la Revue des Ressources. Available at: http ://www. larevuedesressources.org/theorie-de-la-derive,038.html (accessed 10 June 2017).

Elkin L. (2016) Flâneuse: Women Walk the City in Paris, New York: Farrar, Straus and Giroux.

Flâneuse : Women Walk the City in Paris, New York, Tokyo, Venice, Farrar, Straus \& Giroux.

Foucault M. (1984) Des espaces autres. Architecture, Mouvement, Continuité, no 5, pp. 46-49.

Giddens A. (1991) The Consequences of Modernity, Cambridge: Polity Press.

Grotta M. (2015) Baudelaire's Media Aesthetics: The Gaze of the Flâneur and 19th Century Media, New York: Bloomsbury.

Merzeau L. (2014) Le flâneur impatient. Médium, Rythmes, no 41, pp. 20-29.

Nuvolati G. (2009) La flâneur dans l'espace urbain. Géographie et cultures, no 70, pp. 7-20.

Park R. E. (1915) The City: Suggestions for the Investigation of Human Behavior in the

Urban Environment. American Journal of Sociology, vol. 20, no 5, pp. 577-612.

Ricoeur P. (1998) Architecture et narrativité. Urbanisme, no 303, pp. 44-51.

Ricoeur P. (2000) Propos d'un flâneur. Diagonal, no 141, pp. 11-13.

Rioux Soucy L.-M. (2016) De la flânerie comme acte de résistance. Le Devoir. Available at: http ://www.ledevoir.com/societe/actualites-en-societe/464778/un-hiver-avec-felix-leclerc-de-la-flanerie-comme-acte-de-resistance (accessed 10 June 2017).

Shin J. (2014) Le flâneur postmoderne: entre solitude et être-ensemble, Paris: CNRS.

Simmel G. (2002) The Metropolis and Mental Life. The Blackwell City Reader (eds. G. Bridge, S. Watson), Oxford : Wiley-Blackwell, pp. 11-19.

Wrigley R. (2016) Flâneur. Oxford Art Journal, vol. 39, no 2, pp. 267-284. 


\title{
Фланер - читатель современного города
}

\author{
Анна Борисенкова \\ Исследователь, лектор Университета Сен-Бонифаса, Виннипег \\ Старший научный сотрудник Центра фундаментальной социологии Национального исследовательского \\ университета «Высшая школа экономики» \\ Адрес: avenue de la Cathédrale, 200, Winnipeg, Canada $\mathrm{R}_{2} \mathrm{H}$ oH7 \\ E-mail:ann.borisenkova@gmail.com
}

Тема любителя уличной жизни, наблюдателя, городского бродяги и скитальца впервые появляется в работе Эдгара Аллана По «Человек толпы». Однако термин «фланер» закрепился в гуманитарных науках благодаря Шарлю Бодлеру, и впоследствии, - эссе Вальтера Беньямина, к котором фланер фигурирует в качестве центральной фигуры современной жизни. Недавние исследования в социальных и гуманитарных науках в значительной степени пересматривают роль фланера и городского явления фланирования. К описанию фланера в основном применимы два критерия: степень вовлеченности фланера в жизнь города и его способность трансформировать смысловую структуру городского пространства. Согласно данным критериям можно выделить фланеров-исследователей и фланеров, участвующих в политической жизни города. С другой стороны, мы можем отметить типы фланера, которые пассивно наблюдают происходящее или являются потребителями, бесцельно прогуливающимся по современным торговым центрам. Цель статьи - проанализировать эволюцию понятия фланирования и рассмотреть альтернативный подход к данному понятию. Теоретической основой альтернативного подхода является герменевтическая программа Поля Рикера, представляющая фланера в качестве активного читателя и актора-пользователя публичных мест города, а также создателя коллективной памяти.

Ключевые слова: фланер, городское пространство, публичное пространство, читатель, память, нарратив 